\title{
Reconfigurability Evaluation of Multifunctional Intelligent Boom Sprayer Based on Fuzzy Comprehensive Evaluation
}

\author{
Luqiang Ma $\mathbb{D}^{\text {, }}$, Youlin Xu $\mathbb{D}$, Jiaqiang Zheng, and Xiang Dai \\ College of Mechanical and Electronic Engineering, Nanjing Forestry University, Nanjing 210037, China \\ Correspondence should be addressed to Youlin Xu; youlinxu@njfu.edu.cn
}

Received 9 September 2019; Revised 20 November 2019; Accepted 31 January 2020; Published 17 March 2020

Academic Editor: Leonid Shaikhet

Copyright $\odot 2020$ Luqiang Ma et al. This is an open access article distributed under the Creative Commons Attribution License, which permits unrestricted use, distribution, and reproduction in any medium, provided the original work is properly cited.

\begin{abstract}
Multifunctional intelligent boom sprayer is a development direction of boom sprayers, and it could satisfy needs of precision and variable spraying and achieve aims of sustainable development of pesticide spraying, ecological protection, and efficient plant protection by reconfiguring its structures. Therefore, the evaluation of multifunctional intelligent boom sprayer reconfigurability has a role in improving product reconfigurability while reducing product function redundancy and production costs and reducing product function redundancy and production cost. In order to comprehensively evaluate the reconfigurability of a multifunctional intelligent boom sprayer, this paper established an evaluation index system and realized the evaluation process by using fuzzy comprehensive evaluation, analytic hierarchy process, and validity test. The evaluation result would avail to directly and effectively guide product optimization and upgrade process in accordance with quantitatively analyzed evaluation indexes of intelligent boom sprayer reconfigurability by using mathematical methods such as analytic hierarchy process and validity test in evaluation process. The index system was formed from six components, which were key design information, quality, cost, efficiency, intelligence, and operational capability. Index weights were calculated by the analytic hierarchy process. Finally, a multifunctional shiftable boom intelligent sprayer was evaluated for product reconfigurability improvements by using the evaluation method. Reconfigurability evaluation result of the multifunctional shiftable boom intelligent sprayer belonged to "good" evaluation interval by the calculation of formulas. There was further room to improve product reconfigurability, as the membership degree in the "good" evaluation interval was below 0.5 . The study could provide a scientific basis for improving the reconfigurability, technical performance, and operation quality of products in intelligent boom sprayer design. In addition, it may be possible to decrease the arbitrary nature of subjective design factors and support economic and ecological goals when businesses upgrade products.
\end{abstract}

\section{Introduction}

Reconfigurable system is the one that can change its configuration dynamically in response to changes in conditions in order to achieve operational requirements [1]. Reconfigurable systems can help to improve the versatility and adaptability of agricultural machinery in complex, diverse operating environments. With the adjustment of the scale structure of farmland in China, the proportion of boom sprayers used to prevent and control large-scale crop pests and diseases has increased to meet pest control and defoliant spraying needs [2]. Therefore, it is a development trend about boom sprayers, which is to develop an intelligent boom sprayer that can meet the individual needs of farmers, realize the intelligence of boom sprayers, and achieve the requirements of the reconfigurable system. IBS, intelligent boom sprayer, should be able to adapt to the precise, variable pesticide, water, and fertilizer application requirements associated with various working environments and objectives through the reconfiguration of its software/hardware modules. IBS adapts to the requirements of the reconfigurable system and pays attention to the sustainable development of the ecological environment while reducing the total cost of products and improving the economic benefits of the products. There are boom-transformable sprayers, variable spray boom sprayers, profiling sprayers, unmanned sprayers, and so on, belonging to IBS with various degrees of intelligence. Therefore, IBS is to use new intelligent technologies to improve and develop the existing boom sprayers to achieve product reconfiguration. The reconfigurability of 
IBS is a key index to realize its capabilities. The research on the IBS reconfigurable system assessment can provide a new research idea and executable method to evaluate the reconfigurability of IBS and provide technical support for the IBS rapidly improving development.

At present, boom sprayer evaluation is focused primarily on its adaptive evaluation [3] and considers the effects of specific boom sprayer functions [2, 4-10]. In contrast, there are relatively few studies that consider boom sprayer reconfigurability and engineering.

Therefore, this paper seeks to develop an IBS reconfigurability evaluation index that considers the external application effects, internal structural engineering, product economics, etc. to provide a comprehensive, objective, and scientific evaluation system. Meanwhile, the evaluation index system should achieve the requirement which can help people in different fields related to IBS, especially product users, to participate in the evaluation process, so that the product can adapt to market changes. The IBS reconfigurability evaluation may be achieved by applying fuzzy comprehensive evaluation to the evaluation index system. In the process, the index weight could be decided by using the analytic hierarchy process. We would use a validity test to ensure the validity of the evaluation results. The evaluation results could clearly reflect the probability value of reconfigurability evaluation in different result domains by using the mathematical methods such as analytic hierarchy process and validity test of the evaluation process. The study can provide a scientific basis for improving the reconfigurability of IBS and also provide directing guidance for enterprises to optimize the product process. It could avoid fuzzy or qualitative evaluation results and lead designers to optimize and upgrade a product module in ambiguous judgement environment. Finally, it is proposed to establish an IBS reconfigurability evaluation system for evaluating and improving products reconfigurability.

\section{Analysis of Reconfigurability Evaluation Index on IBS}

In order to comprehensively and scientifically evaluate the reconfigurability of IBS, the IBS reconfigurability evaluation index system needs to be constructed for researching the scientific and reasonable evaluation method. In the process, the validity test and index weight determination would be studied.

\subsection{Business Impact Analysis Model of Brownfield Process.} BfP, Brownfield Process, is a product development approach for modular product families that focuses on product configuration and emphasizes reuse of existing resources and standardized applications while rationalizing product diversity [11]. BfP considers key engineering concepts comprehensively while other design methods consider them only partially or in various combinations. These key engineering concepts include partitioning logic, set of modules, interfaces, architecture, and configuration knowledge. These are indispensable for modular product family development
[12]. BIA, business impact analysis, describes the interrelationships between key engineering concepts, supports the guidelines and mechanisms for the rationalization of manufacturing industrial product diversity and general manufacturing processes, and reflects the maximum benefit evaluation of modular systems in BfP [11]. In order to reflect the completeness of key product design information, the IBS reconfigurability evaluation index system was constructed based on BIA.

\subsection{Construction of IBS Reconfigurability Evaluation Index} System. The evaluation index system comprehensively reflects the IBS reconfigurability based on the principles of comprehensiveness, comparability, scientificity, and practicability. The IBS reconfigurability evaluation index was constructed by combing with the relevant standards of boom sprayers [13-16] and the connotation of IBS and the BIA model, which is based on the evaluation index of the existing boom sprayers. It has focused on standardization and resource reuse. The reconfigurability evaluation indexes also focused on the concept of standardization and resource reuse in enterprises. The IBS reconfigurability evaluation index architecture model was divided into three layers. The target layer was IBS reconfigurability evaluation. The criterion layer (first-level index) included six components, key design information (partitioning logic, set of modules, interfaces, architecture, and configuration knowledge), quality, cost, benefit, intelligence, and operational capability. The relevant program layer content (secondary-level index) is shown in Figure 1, and the relevant technical indexes are explained in Table 1.

\subsection{Fuzzy Comprehensive Evaluation Process and Analytic} Hierarchy Process. In systems engineering, fuzzy comprehensive evaluation is a practical method designed to quantify empirical results in complex systematic evaluation conditions by combining multiple indexes with quantitative and qualitative results [13]. Index weights are determined via an analytic hierarchy process. The evaluation process is as follows [18-26]:

(1) $F=\left\{F_{1}, F_{2}, \ldots, F_{i}\right\}$, and the established factor domains are a set of evaluation indexes which indicate that $F_{i}$, the first-level indexes, needs to be evaluated. In this context, $i=1,2, \ldots, n$. At the same time, the secondary indexes included in each $F_{i}$ are represented by $C_{i j}$, where $j=1,2, \ldots, m$.

(2) $E=\left\{E_{1}, E_{2}, \ldots, E_{k}\right\}$ represents the established result domains, which includes a set of evaluation levels. In order to avoid excessive invalidation of the evaluation grade division granularity, $E=\{$ very good, good, general, bad $\}$ is taken as the result domain for IBS reconfigurability evaluation and $k=1,2, \ldots, 4$. The "very good" percentile interval is $[90,100]$, the "good" percentile interval is $[75,90]$, the "general" percentile interval is $[60,75]$, and the "bad" percentile interval is $[0,60][25]$. 


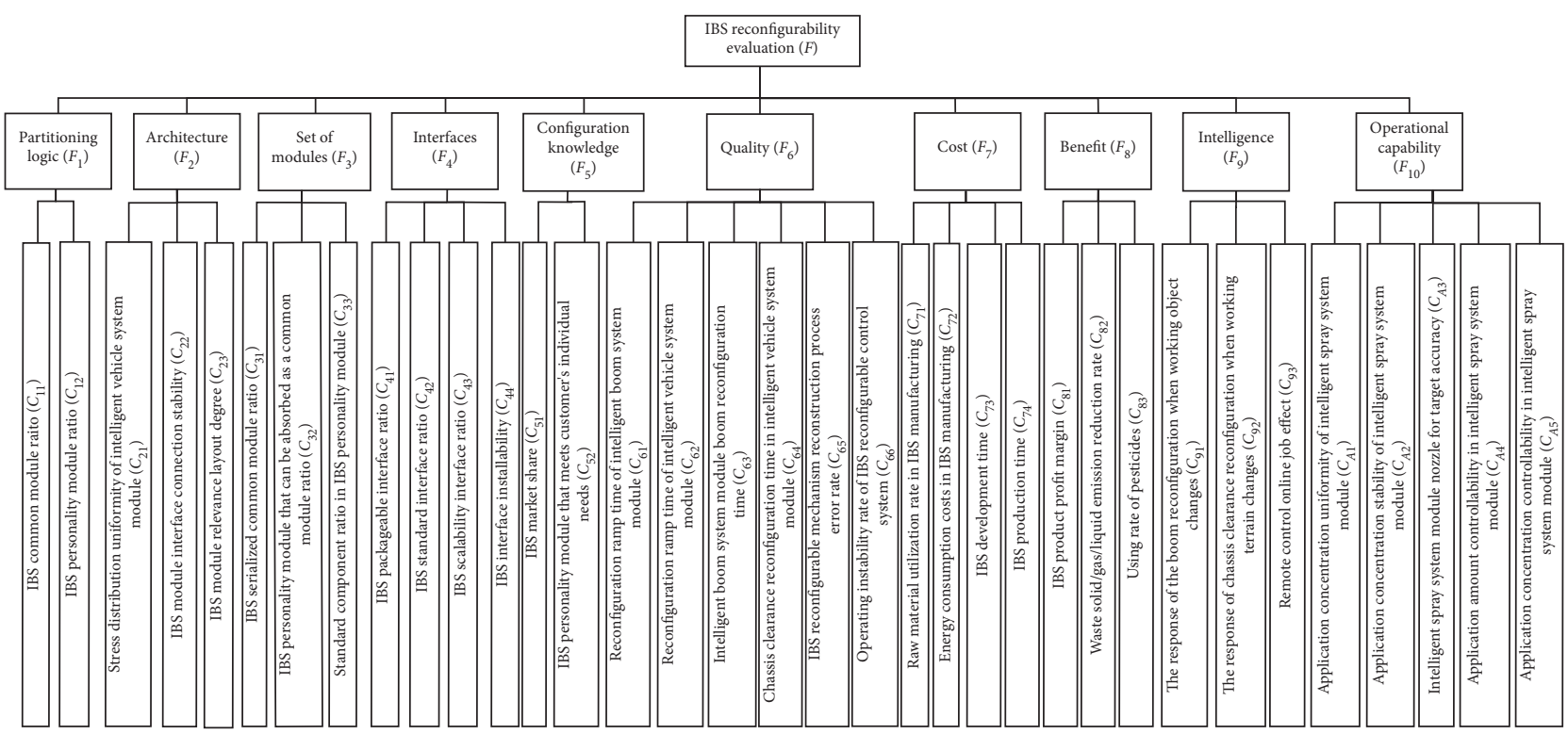

Figure 1: Evaluation index system. The evaluation index system includes target, criterion, and program layers.

(3) $R=\left\{R_{1}, R_{2}, \ldots, R_{i}\right\}^{T}$ is the established membership matrix. $r_{i j k}=\left(E_{i j k} / n\right)$ in $R_{i}=\left(r_{i j 1}, r_{i j 2}, \ldots, r_{i j k}\right)$, membership degree, is the mean of the probability that the $n$ evaluation subjects achieve rating $E_{k}$ in terms of $C_{i j}$ and $\sum_{k=1}^{4} r_{i j k}=1$, where $n$ is the number of experts.

(4) $T$ is the evaluation index weight and is established using analytic hierarchy process. $A$ is the comparison matrix and is established by applying Delphi method to the evaluation system hierarchy model. The scoring rules are determined in accordanc with the importance of the former relative to the latter. A score of 1 indicates the same importance, a score of up to 9 indicates that the former is more important than the latter, and a score of less than 1 indicates that the latter is more important than the former. $a_{\text {st }}$ is the score value in $A . W_{i}=\left(W_{1}, W_{2}, \ldots, W_{s}\right)^{T}$, the hierarchical single sort result, is determined by equations (1) and (2), the maximum eigenvalue $\lambda_{\max }$ is determined by equation (3), and the consistency check is determined by equations (4) and (5).

$$
\begin{aligned}
W_{s} & =\frac{w_{s}}{\sum_{s=1}^{j}\left(w_{s}\right)}, \\
w_{s} & =\sqrt[j]{\prod_{t=1}^{j} a_{s t}} s=1,2, \ldots, j, \\
\lambda_{\max } & =\frac{1}{j} \sum_{s=1}^{j} \frac{(A W)_{s}}{W_{s}}, \\
\mathrm{CI} & =\frac{\lambda_{\max }-j_{\max }}{j_{\max }-1},
\end{aligned}
$$

$$
\mathrm{CR}=\frac{\mathrm{CI}}{\mathrm{RI}}<0.1
$$

$\mathrm{RI}$ is the average random consistency index. $\mathrm{CI}$ is the consistency index. CR is the consistency ratio. If $\mathrm{CI}=0$, the comparison matrix is completely consistent; if $\mathrm{CI} \neq 0$, but equation (5) is satisfied, the matrix has satisfactory consistency, and the comparison matrix is acceptable. If equation (5) is not satisfied, the comparison matrix must be modified until equation (5) becomes satisfied. RI can be selected according to Table 2 [16]. $j_{\max }$ is the total number of secondary indexes under the first-level indexes $F_{i}$. Finally, a consistency check of the resulting total hierarchical ranking is performed such that the inspection process is consistent with the single-level consistency test. After passing the test, the evaluation index weight $T$, $T=\left(T_{1}, T_{2}, \ldots, T_{i}\right)$, can be obtained. When calculating the weight of the first-level indexes, $j$ takes the value $i$, and $j_{\max }$ is the maximum value of $i$ in equations (1) $-(4)$.

(5) Fuzzy comprehensive evaluation includes evaluation of target level, first-level index, and second-level index. First, the secondary index levels should be evaluated, and the percentage of the secondary indexes $V_{C_{i j}}$ should be determined by equation (6). $E_{\text {mid }}$ is the mean value of percentile intervals in $E$. Second, the comprehensive evaluation vector $B_{i}$ of the first-level index is obtained using equation (7). Finally, the comprehensive evaluation vector $B$ of the target level is obtained using equation (8). The comprehensive evaluation vector identifies indexes that need to be improved. Corresponding improvement plans can be formulated to avoid blind optimization and upgrade of products and improve the reconfigurability and quality of IBS. 


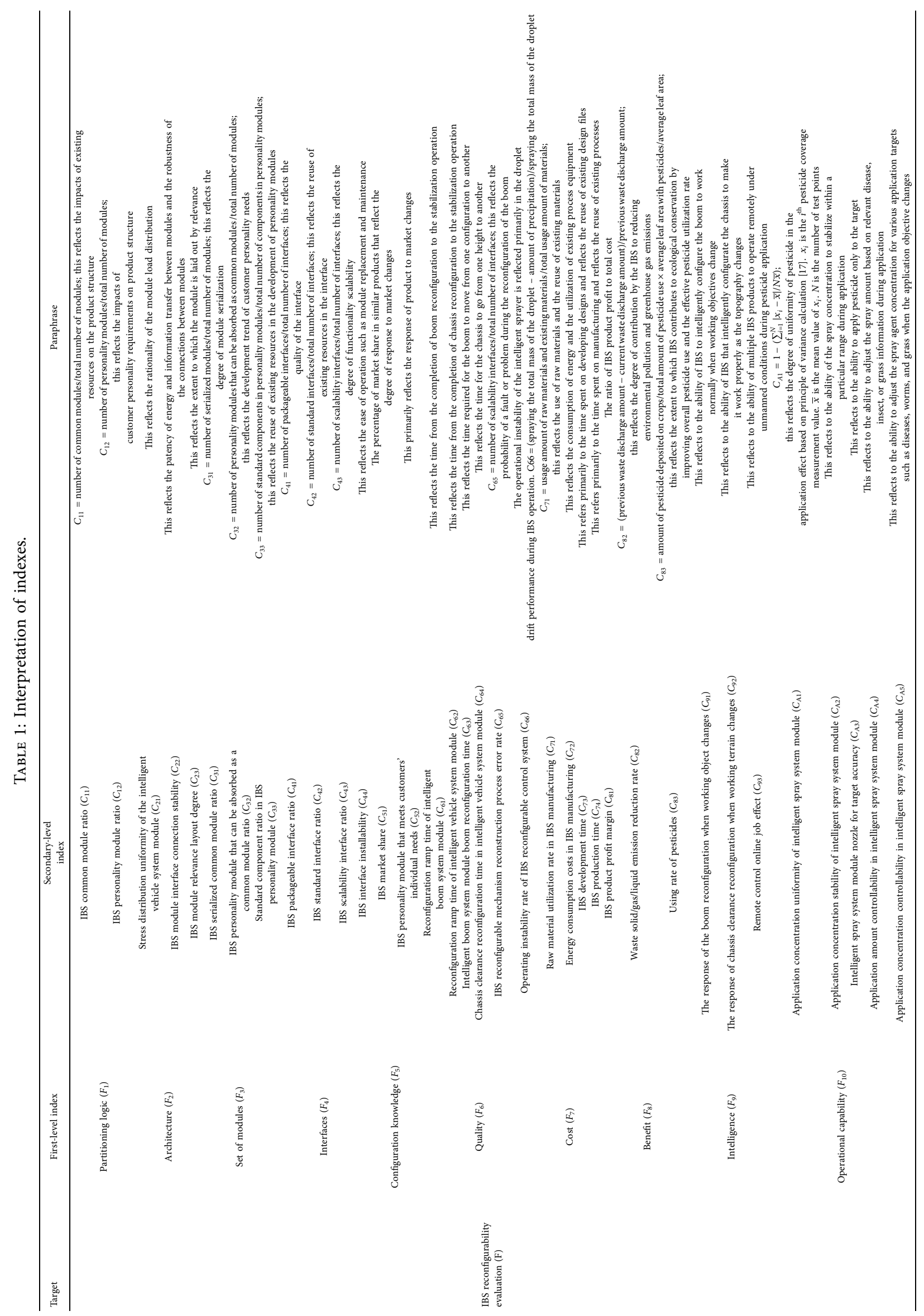


TABLE 2: Average random index [16].

\begin{tabular}{|c|c|c|c|c|c|c|c|c|c|c|c|c|c|c|}
\hline$n$ & 1 & 2 & 3 & 4 & 5 & 6 & 7 & 8 & 9 & 10 & 11 & 12 & 13 & 14 \\
\hline RI & 0 & 0 & 0.52 & 0.89 & 1.12 & 1.26 & 1.36 & 1.41 & 1.46 & 1.49 & 1.52 & 1.54 & 1.56 & 1.58 \\
\hline
\end{tabular}

$$
\begin{aligned}
V_{C_{i j}} & =R_{i j} \cdot E_{\mathrm{mid}}^{T}, \\
B_{i} & =W_{i}^{T} \cdot R_{i}, \\
B & =T \cdot\left(B_{1}, B_{2}, \ldots, B_{i}\right)^{T} .
\end{aligned}
$$

2.4. Validity Test Process. The maximum membership degree principle, which is typically followed during comprehensive evaluation vector processing, may be invalid under certain conditions [26]. Therefore, some mathematics researchers have proposed the maximum membership validity test method, as shown in the following equation $[23-25,27]$ :

$$
\alpha=\frac{(n \beta-1)}{(2 \gamma(n-1))} .
$$

In equation (9), $\alpha$ is the result of validity test. If $\alpha=+\infty$, the maximum membership degree principle is completely valid; if $1 \leq \alpha<+\infty$, the maximum membership degree principle is highly effective; if $0.5 \leq \alpha<1$, the maximum membership degree principle is generally effective; if $0 \leq \alpha<0.5$, the maximum membership degree principle is minimally effective; and if $\alpha=0$, the maximum membership degree principle is completely invalid. In this equation, $n$ is the elements number of $E ; \beta$ is the maximum membership degree in $B$; and $\gamma$ is the maximum membership degree after $\beta$ is removed from $B$.

The validity test is then quantified because the valuation result cannot be judged accurately when $\alpha<0.5$ or $\alpha=0$. The steps of quantification process are as follows [25]:

(1) The highest score, $S_{H}$, and lowest score, $S_{L}$, from the final comments are calculated, as shown in the following equations:

$$
\begin{gathered}
S_{H}=B \cdot E_{K H}, \\
S_{L}=B \cdot E_{K L},
\end{gathered}
$$

where $E_{K H}$ and $E_{K L}$ are the maximum and minimum in each percentile interval $E_{K}$.

(2) The interval length $L$ is calculated as shown in the following equation:

$$
L=S_{H}-S_{L}
$$

(3) The probability of the evaluation results in each percentile interval, $P_{K}$, is calculated as shown in equation (13). $L_{K}$ evaluation result interval length for each percentile interval $E_{K}$.

$$
P_{K}=\frac{L_{K}}{L}
$$

(4) The evaluation result based on $\max P_{K}$ is determined.

2.5. Index Weight Determination. The weights in the evaluation index system are calculated by using the content of process (4) in Section 2.3. A group of 13 researchers [27], who understood the concepts of IBS and reconfigurable theory participated in the index weight determination process by referencing the Delphi method. The first-level factors index weights could be confirmed in accordance with these researchers' opinions and process (4) in Section 2.3, as shown in Table 3.

In this manner, weights of other indexes were obtained. The calculation results are shown in Table 4.

\section{Case Study}

The multifunctional shiftable boom intelligent sprayer developed by the research group is a typical IBS that can meet the target operation requirements of field pest control for field crops, hedge crops, fruit trees, and forests. It can reconfigure the tractor chassis structure to meet plant protection requirements for various crop types and plant heights via dynamic adjustment of product operation parameters within a given range in response to changes in objectives or operating conditions. The multifunctional shiftable boom intelligent sprayer can solve the problem of mechanization, specialization, and intelligence of pest control in a variety of business models with limited scale of agricultural and forestry production land. It can perform multiple agricultural production functions to maximize product use efficiency. The multifunctional shiftable boom intelligent sprayer is shown in Figure 2 [28, 29]. The maximum spray width of the prototype is $9 \mathrm{~m}$, the " $U$ " frame has dimensions of $1.5 \times 1.5 \mathrm{~m}$, and the maximum lifting height is $3 \mathrm{~m}$. At the same time, the prototype can realize shrinking and transporting state of boom. The boom has three types of working status, including shapes of “-,, "U," and " $\cap$." The prototype control system enables intelligent target spraying [30-32] and dynamically controls the number of nozzles activated in accordance with the presence of crops. The presence of crops could be identified by using an infrared optoelectronic sensor on the nozzles. The spraying system can also adjust the duty cycle of the solenoid valve to maintain spray system flow stability and pressure in order to meet agricultural production requirements. The working speed of the prototype is $2 \sim 6 \mathrm{~km} / \mathrm{h}$, application rate is $350 \mathrm{~L} / \mathrm{ha}$, comprehensive utilization rate of pesticide is $\geq 30 \%$, pressure is $0.3 \mathrm{Mpa}$, and flow is $2 \mathrm{~L} / \mathrm{min} /$ nozzle. The 
TABLE 3: The scoring of first-level factors.

\begin{tabular}{|c|c|c|c|c|c|c|c|c|c|c|c|c|}
\hline$F$ & $F_{1}$ & $F_{2}$ & $F_{3}$ & $F_{4}$ & $F_{5}$ & $F_{6}$ & $F_{7}$ & $F_{8}$ & $F_{9}$ & $F_{10}$ & $w_{s}$ & $W_{s}$ \\
\hline$F_{1}$ & 1 & 1 & 1 & 1 & 1 & $1 / 4$ & $1 / 3$ & $1 / 4$ & $1 / 3$ & $1 / 4$ & 0.5296 & 0.043352625 \\
\hline$F_{2}$ & 1 & 1 & 1 & 1 & 1 & $1 / 4$ & $1 / 3$ & $1 / 4$ & $1 / 3$ & $1 / 4$ & 0.5296 & 0.043352625 \\
\hline$F_{3}$ & 1 & 1 & 1 & 1 & 1 & $1 / 4$ & $1 / 3$ & $1 / 4$ & $1 / 3$ & $1 / 4$ & 0.5296 & 0.043352625 \\
\hline$F_{4}$ & 1 & 1 & 1 & 1 & 1 & $1 / 4$ & $1 / 3$ & $1 / 4$ & $1 / 3$ & $1 / 4$ & 0.5296 & 0.043352625 \\
\hline$F_{5}$ & 1 & 1 & 1 & 1 & 1 & $1 / 4$ & $1 / 3$ & $1 / 4$ & $1 / 3$ & $1 / 4$ & 0.5296 & 0.043352625 \\
\hline$F_{6}$ & 4 & 4 & 4 & 4 & 4 & 1 & 2 & 1 & 2 & 1 & 2.2974 & 0.188063294 \\
\hline$F_{7}$ & 3 & 3 & 3 & 3 & 3 & $1 / 2$ & 1 & 2 & 1 & $1 / 2$ & 1.6161 & 0.13229263 \\
\hline$F_{8}$ & 4 & 4 & 4 & 4 & 4 & 1 & $1 / 2$ & 1 & $1 / 2$ & 1 & 1.7411 & 0.142525028 \\
\hline$F_{9}$ & 3 & 3 & 3 & 3 & 3 & $1 / 2$ & 1 & 2 & 1 & $1 / 2$ & 1.6161 & 0.13229263 \\
\hline$F_{10}$ & 4 & 4 & 4 & 4 & 4 & 1 & 2 & 1 & 2 & 1 & 2.2974 & 0.188063294 \\
\hline \multicolumn{3}{|c|}{$\lambda_{\max }=10.21247659$} & \multicolumn{3}{|c|}{$\mathrm{CI}=0.023609$} & \multicolumn{4}{|c|}{$\mathrm{RI}=1.49$} & \multicolumn{3}{|c|}{$\mathrm{CR}=0.015845<0.1$} \\
\hline
\end{tabular}

TABLE 4: The weights of evaluation indexes on IBS.

\begin{tabular}{|c|c|c|c|c|c|c|c|c|c|c|}
\hline $\begin{array}{l}\text { Index } \\
\text { code }\end{array}$ & $F_{1}$ & $F_{2}$ & $F_{3}$ & $F_{4}$ & $F_{5}$ & $F_{6}$ & $F_{7}$ & $F_{8}$ & $F_{9}$ & $F_{10}$ \\
\hline & 0.0433526 & 0.04335262 & 0.0433526 & 0.0433526 & 0.0433526 & 0.188063294 & 0.132293 & 0.142525 & 0.132293 & 0.188063294 \\
\hline$C_{11}$ & 0.7500054 & & & & & & & & & \\
\hline$C_{12}$ & 0.2499946 & & & & & & & & & \\
\hline$C_{21}$ & & 0.2000127 & & & & & & & & \\
\hline$C_{22}$ & & 0.39999365 & & & & & & & & \\
\hline$C_{23}$ & & 0.39999365 & & & & & & & & \\
\hline$C_{31}$ & & & 0.296965 & & & & & & & \\
\hline$C_{32}$ & & & 0.5396151 & & & & & & & \\
\hline$C_{33}$ & & & 0.1634198 & & & & & & & \\
\hline$C_{41}$ & & & & 0.4929715 & & & & & & \\
\hline$C_{42}$ & & & & 0.1954152 & & & & & & \\
\hline$C_{43}$ & & & & 0.1161981 & & & & & & \\
\hline$C_{44}$ & & & & 0.1954152 & & & & & & \\
\hline$C_{51}$ & & & & & 0.6666667 & & & & & \\
\hline$C_{52}$ & & & & & 0.3333333 & & & & & \\
\hline$C_{61}$ & & & & & & 0.035963014 & & & & \\
\hline$C_{62}$ & & & & & & 0.035963014 & & & & \\
\hline$C_{63}$ & & & & & & 0.139475789 & & & & \\
\hline$C_{64}$ & & & & & & 0.139475789 & & & & \\
\hline$C_{65}$ & & & & & & 0.324561197 & & & & \\
\hline$C_{66}$ & & & & & & 0.324561197 & & & & \\
\hline$C_{71}$ & & & & & & & 0.125005412 & & & \\
\hline$C_{72}$ & & & & & & & 0.125005412 & & & \\
\hline$C_{73}$ & & & & & & & 0.374994588 & & & \\
\hline$C_{74}$ & & & & & & & 0.374994588 & & & \\
\hline$C_{81}$ & & & & & & & & 0.142848652 & & \\
\hline$C_{82}$ & & & & & & & & 0.428575674 & & \\
\hline$C_{83}$ & & & & & & & & 0.428575674 & & \\
\hline$C_{91}$ & & & & & & & & & 0.428575674 & \\
\hline$C_{92}$ & & & & & & & & & 0.428575674 & \\
\hline$C_{93}$ & & & & & & & & & 0.142848652 & \\
\hline$C_{\mathrm{A} 1}$ & & & & & & & & & & 0.285706207 \\
\hline$C_{\mathrm{A} 2}$ & & & & & & & & & & 0.285706207 \\
\hline$C_{\mathrm{A} 3}$ & & & & & & & & & & 0.142862529 \\
\hline$C_{\mathrm{A} 4}$ & & & & & & & & & & 0.142862529 \\
\hline$C_{\mathrm{A} 5}$ & & & & & & & & & & 0.142862529 \\
\hline
\end{tabular}

minimum and maximum boom heights are $3000 \mathrm{~mm}$ and $600 \mathrm{~mm}$, respectively. MDCO nozzles are used.

A group of 13 researchers evaluated the multifunctional intelligent boom sprayer reconfigurability by using the IBS evaluation index system and process (4) in Section 2.3, for scientifically evaluating and optimizing the multifunctional shiftable boom intelligent sprayer and gradually meeting the IBS requirements. During evaluation, some indexes were determined by the simple testing method or subjective way, such as determination using a stopwatch or manual observation operation. This can aid in further research and aid users who participate in index evaluation. The 


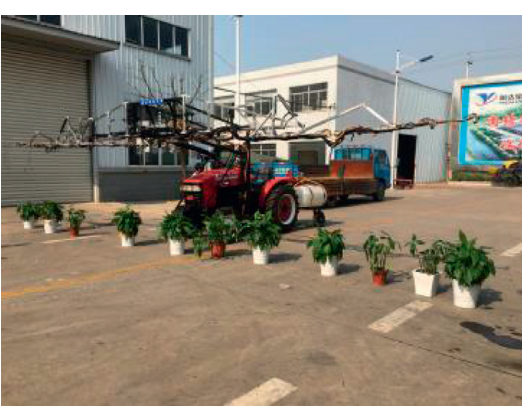

(a)

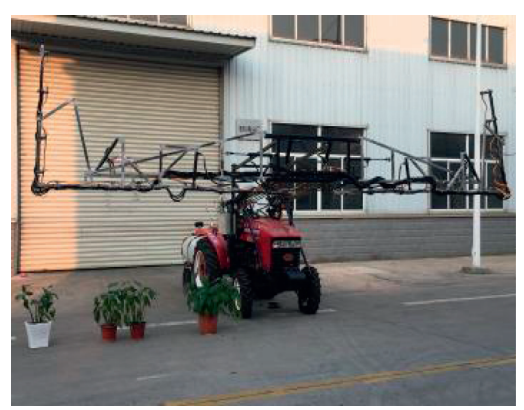

(b)

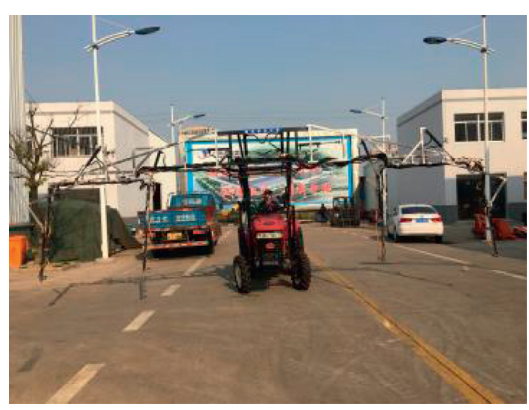

(c)

FIgURE 2: Reconfiguration images of the multifunctional shiftable boom intelligent sprayer. (a) The shape of “-." (b) The shape of "U.” (c) The shape of " $n$."

reconfigurability evaluation result was verified using validity testing.

The results from evaluating all the secondary indexes under $F_{i}$ can be determined using equation (6). The index evaluation results are shown in Figure 3. The evaluation result of the first-level index $F_{i}$ can be obtained using equation (7), and the index evaluation results are shown in Figure 4.

The evaluation result of target-level $F$ can be obtained according to equation (8). Therefore, there was membership degree of $E=$ \{very good, good, general, bad $\}$ of target-level $F$ which was " $0.276955,0.420507,0.203853,0.098685$," respectively. The $\alpha$ value could be determined using validity testing, as shown in the following equation:

$$
\alpha=\frac{(4 \times 0.420507-1)}{(2 \times 0.276955 \times(4-1))}=0.41 .
$$

According to $\alpha=0.41<0.5$, the maximum membership degree principle was minimally effective. The evaluation result of target-level $F$ should be judged accurately using the quantification process in Section 2.4. Thus, $S_{H}, S_{L}$, and $L$ could be obtained using equations (10)-(12), as shown in the following equations:

$$
\begin{aligned}
& S_{H}=\left(\begin{array}{llll}
0.276955 & 0.420507 & 0.203853 & 0.098685
\end{array}\right) \\
& \cdot\left(\begin{array}{c}
100 \\
90 \\
75 \\
60
\end{array}\right)=86.75121 \\
& S_{L}=\left(\begin{array}{llll}
0.276955 & 0.420507 & 0.203853 & 0.098685
\end{array}\right) \\
& \cdot\left(\begin{array}{c}
90 \\
75 \\
60 \\
0
\end{array}\right)=68.69516 \text {, } \\
& L=S_{H}-S_{L}=18.05605 .
\end{aligned}
$$

The $S_{H}$ and $S_{L}$ values were, respectively, located in good and general evaluation intervals. The boundary between good and general evaluation intervals occurs at 75 .
Therefore, $L_{K}$ could be calculated using equations (18)-(20). $P_{K}$ could be calculated using equation (13), as shown in equations (21)-(23).

$$
\begin{aligned}
& L_{2}=S_{H}-75=86.75121-75=11.75121, \\
& L_{3}=75-S_{L}=75-68.69516=6.304836 \\
& L_{1}=L_{4}=0 \\
& P_{2}=\frac{L_{2}}{L}=\frac{11.75121}{18.05605}=0.650819 \\
& P_{3}=\frac{L_{3}}{L}=\frac{6.304836}{18.05605}=0.349181 \\
& P_{1}=P_{4}=0 .
\end{aligned}
$$

\section{Results and Discussion}

The probability values of different level indexes in different result domains could be obtained through the formula calculation from the fuzzy comprehensive evaluation, analytic hierarchy process, and validity test. Technicians can use these probability values to determine whether to perform further analysis of influencing factors. Reconfigurability evaluation result of multifunctional shiftable boom intelligent sprayer was belonged to good evaluation interval, owing to the $\max P_{K}=P_{2}$. However, the membership degree within the "good" evaluation interval was below 0.5. Although the product achieved some reconfigurability, there was room for improvement.

In Figure 4, the evaluation result of $F_{9}$ was in the poor evaluation interval. When the product is optimized and upgraded, the index and its corresponding secondary-level indexes should be the areas of focus. The evaluation results of $F_{5}$ and $F_{6}$ fall within the "bad" interval. This type of index and its secondary indexes should be secondary concerns when optimizing and upgrading products. Although the evaluation results of $F_{1}, F_{2}, F_{3}, F_{4}, F_{7}$, and $F_{10}$ were located in the "good" interval, their membership degree within the "good" evaluation interval was below 0.5. In addition, other first-level indexes have membership degree above 0.5 in 


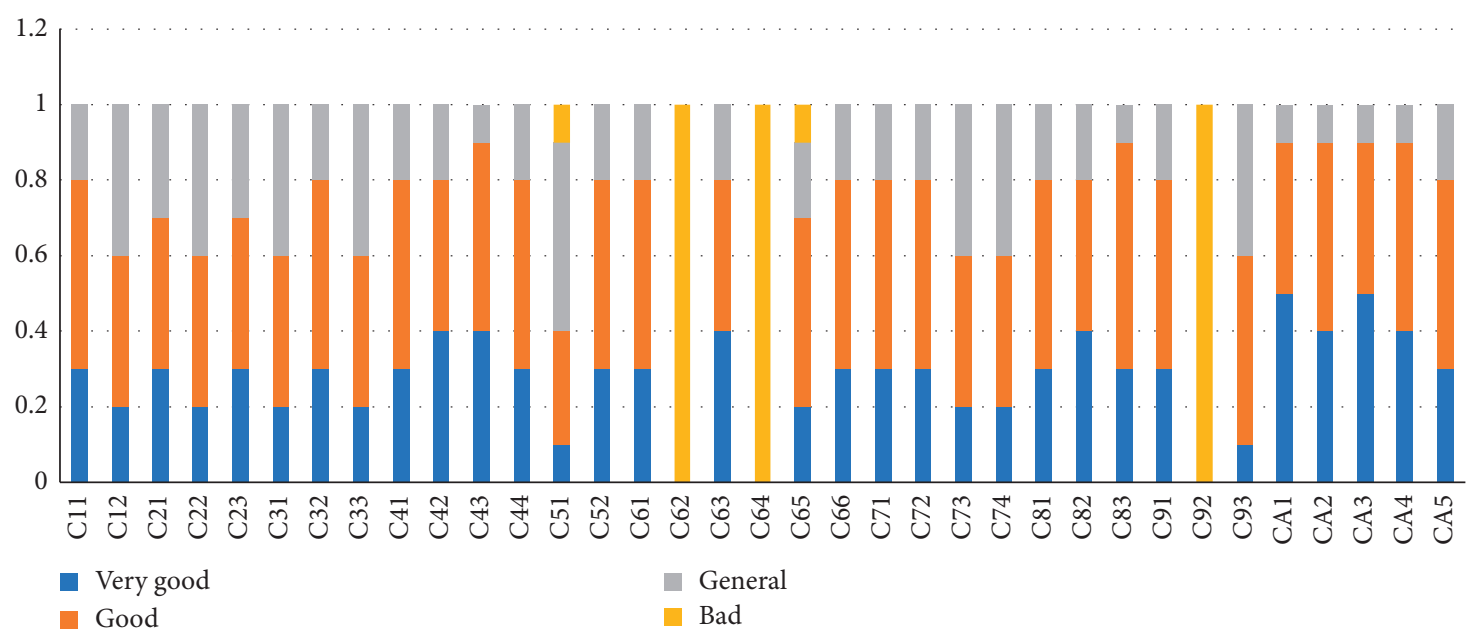

Figure 3: Evaluation results of the secondary indexes. The colour bars represent the evaluation results of secondary-level indexes in the result domain.

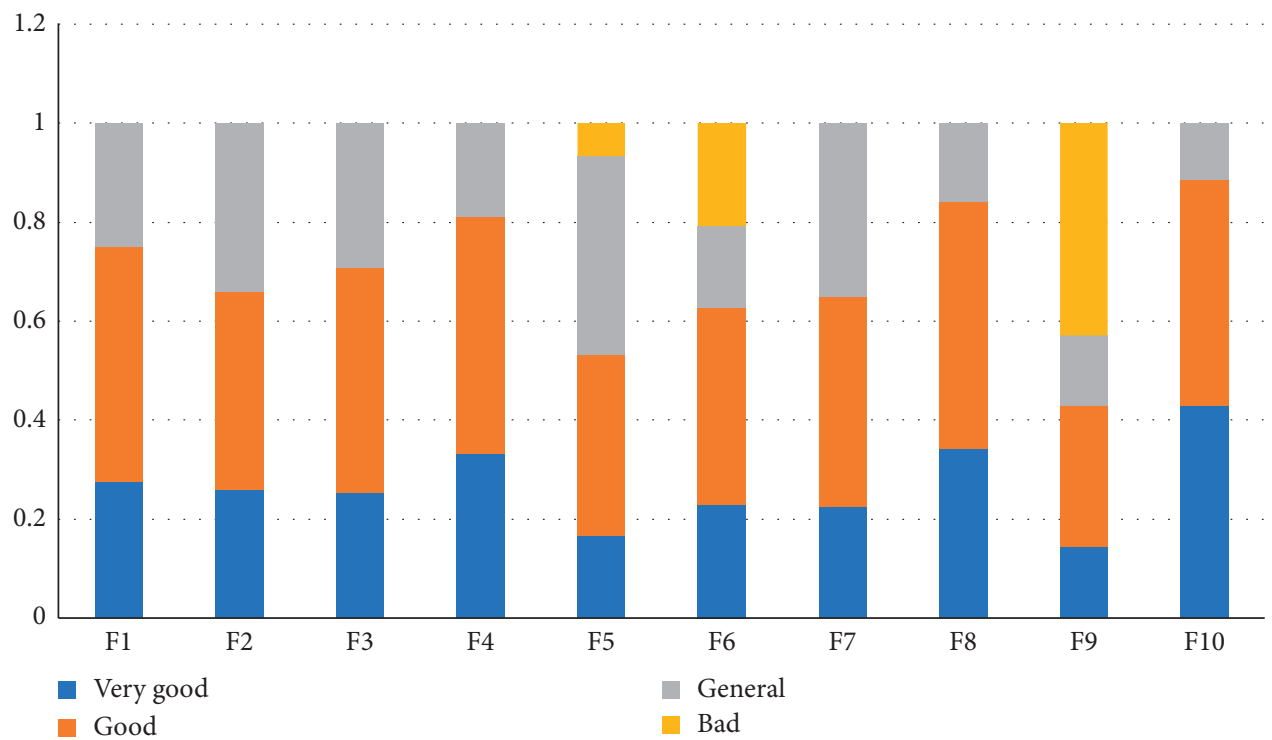

Figure 4: Evaluation results of the first-level indexes. The colour bars represent the evaluation results of first-level indexes in the result domain.

"good" evaluation interval. These can be appropriately optimized and improved in accordance with the existing resource conditions and market changes of the enterprise. Enterprises can formulate product optimization plans to meet market requirements via enterprise resource optimization and reconfiguration using analysis of these indexes. In the next evaluation cycle, the improved product would be evaluated and compared to previous evaluation results. From the comparison results, the customer satisfaction of the optimized product, the market's changing trend, and the applicability of the index system are analyzed. The evaluation cycle and indicator system revision cycle can be determined based on the product market cycle. Furthermore, this can lead to construction of a product periodic optimization mechanism to meet the requirements of market changes.
In Figure 3, the evaluation results of $C_{62}, C_{64}$, and $C_{92}$ were located in the "bad" interval and their membership degree was 1 . These three indexes should be prioritized for optimization, when optimizing its first-level index. Among the secondary indexes, the chassis-related indexes should be fully considered, when the product is optimised and upgraded, for improving the reconfigurability of the chassis. At the same time, it can be seen from $C_{51}$ and $C_{65}$ that product promotion should be strengthened. The secondary level-index is a further reflection of the first-level index. The secondary-level indexes could more accurately reflect the problems of products to assist designers in making optimization plans. And the first-level indexes and its secondarylevel indexes should be implemented simultaneously during periodic product optimization. 
Excessive pursuit of product advances should be avoided and both the enterprise and market development changes should be focused. The index evaluation results can provide decision support data for research and enterprise $R \& D$ personnel in product development and optimization. According to the evaluation of multifunctional intelligent boom sprayer, different optimization and improvement schemes can be formulated to reduce the interference of subjective factors on product optimization schemes and provide scientific basis for more scientific and objective guidance of product optimization. Therefore, this will assist $\mathrm{R} \& \mathrm{D}$ personnel determining design directions in the process of researching or optimizing products. It also reduces unnecessary product development and optimization waste. The index system effectiveness was verified by the site testing of the multifunctional intelligent boom sprayer. The index systems basically expressed various aspects of the multifunctional intelligent boom sprayer.

\section{Conclusion}

Evaluating IBS reconfigurability allows one to scientifically guide IBS reconfigurability and provides direct product optimization guidance. The IBS reconfigurability evaluation index system was constructed using the BIA model. A fuzzy comprehensive evaluation method was used to evaluate the process of the evaluation index system, and the weight of the index is determined by the hierarchical analysis method. The evaluation result was verified by the validity test.

As a typical IBS, the multifunctional shiftable boom intelligent sprayer developed by the research group was processed using the evaluation system to obtain the comprehensive evaluation result and evaluation results of each individual index. These results intuitively illuminated the overall product and all aspects of the indexes, thus providing a basis for designers to improve the product. The index system effectiveness was verified via the case study. The above research provides an objective, scientific, and complete evaluation method for evaluating IBS reconfigurability and supporting the achievement of corporate green manufacturing goals.

In future, the multifunctional shiftable boom intelligent sprayer would be improved based on this evaluation method. A few IBS would be produced for more researchers to participate in the evaluation process to improve the index systems. It is necessary to create a schedule and plan to update the index system and weights regularly to ensure index system effectiveness.

\section{Data Availability}

The data used to support the findings of this study are available from the corresponding author upon request.

\section{Conflicts of Interest}

The authors declare no potential conflicts of interest with respect to the research, authorship, and/or publication of this article.

\section{Acknowledgments}

This work was sponsored by the Jiangsu Agricultural Machinery Three New Project of China (NJ2014-11).

\section{References}

[1] Y. M. Cheng and L. Q. Ma, "Reconfigurable product systems: a new strategy on product," in Proceedings of the Advances in Communication Technology-2011 3rd World Congress in Applied Computing, Computer Science, and Computer Engineering, vol. 3, pp. 602-607, Newark: Information Engineering Research Institute, Kota Kinabalu, Malaysia, July 2011.

[2] S. L. Yuan and S. Xu, "Lightweight design for frame for boom sprayer based on sensitivity analysis and evaluation methods," Machine Design and Research, vol. 33, no. 2, pp. 108-112, 2017.

[3] Y. Gong, X. Zhang, Y. Liu et al., "Comprehensive evaluation method for applicability of plant protection Y. Achinery based on analytic hierarchy process," Transactions of the Chinese Society for Agricultural Machinery, vol. 47, no. 9, pp. 73-78, 2016.

[4] M. T. Zhang and C. L. Zhang, "Weeding speed and effectiveness of spray atomizer with different spray aerators and different models," Plant Protection Technology and Extension, vol. 23, no. 7, pp. 26-27, 2003.

[5] J. M. Miao, J. M. Zhu, and M. J. Hou, "Selection and evaluation of motorized sprayers," Mechanization of Rural Pastoral Areas, vol. 6, pp. 31-32, 2012.

[6] S. K. Thakare, V. V. Saraf, and M. Deshmukh, "Field evaluation of air assisted sleeve boom sprayer," Madras Agricultural Journal, vol. 102, no. 7-9, pp. 273-276, 2015.

[7] M. E. Teske, H. W. Thistle, G. M. Gross et al., "Evaluation of the wake of an agricultural ground sprayer," Transactions of the ASABE, vol. 58, no. 3, pp. 621-628, 2015.

[8] M. E. Teske, H. W. Thistle, T. C. R. Lawton et al., "Valuation of the flow downwind of an agricultural ground sprayer boom," Transactions of the ASABE, vol. 59, no. 3, pp. 839-846, 2016.

[9] M. Glodeanu, C. Vasile, and T. Alexandru, "Evaluation of a air-carrier sprayer provided with spraying boom equipped with deflecting profiles," in Proceedings of the 16th International Multidisciplinary Scientific Geo Conference, vol. 2, pp. 103-110, Albena, Bulgaria, July 2016.

[10] T. T. Ucar, H. E. Ozkan, R. D. Fox, R. D. Brazee, and R. C. Derksen, "Criteria and procedures for evaluation of solids mixing in agricultural sprayer tanks," Transactions of the ASAE, vol. 42, no. 3, pp. 601-607, 1999.

[11] J. Pakkanen, T. Juuti, and T. Lehtonen, "Brownfield Process: a method for modular product family development aiming for product configuration," Design Studies, vol. 45, pp. 210-241, 2016.

[12] J. Pakkanen, "Brownfield process," Ph. D. thesis, Tampere University of Technology, Tampere, Finland, 2015.

[13] GB/T5271.28-2001, Information Technology-Vocabulary-Part 28: Artificial intelligence-Basic Concepts, China Standard Press, Beijing, 2001.

[14] GB/T24677.2-2009, Boom Sprayer-Test Methods, China Standard Press, Beijing, 2010.

[15] GB/T24677.1-2009, Boom Sprayer-Technical Requirements, China Standard Press, Beijing, 2009.

[16] NY/T1925-2010, Technical Specifications of Quality Evaluation for Boom Sprayers in Use, China Agriculture Press, Beijing, 2010. 
[17] W. Qiu, C. Feng, R. J. Han et al., "Design and test of airassisted electrostatics prayer in greenhouse," Research of Agricultural Modernization, vol. 37, no. 5, pp. 1001-1007, 2016.

[18] Y. L. Wang, Systems Engineering, Mechanical Industry Press, Beijing, China, 4th edition, 2008.

[19] L. Q. Ma, "Research on development and design of reconfigurable product system based on axiomatic design," Master thesis, Anhui Polytechnic University, Wuhu, China, 2012.

[20] R. Zhou and A. H. S. Chan, "Using a fuzzy comprehensive evaluation method to determine product usability: a proposed theoretical framework," Work, vol. 56, no. 1, pp. 9-19, 2017.

[21] R. Zhou and A. H. S. Chan, "Using a fuzzy comprehensive evaluation method to determine product usability: a test case," Work, vol. 56, no. 1, pp. 21-29, 2017.

[22] F. Liu and Z. Ma, "Fuzzy comprehensive evaluation method for symmetry degree of mechanical structure symmetry," Transactions of the Canadian Society for Mechanical Engineering, vol. 41, no. 3, pp. 337-353, 2017.

[23] L. Q. Shen, J. Yang, X. Y. Jin et al., "Evaluating the quality of simulation teaching in Fundamental Nursing Curriculum: AHP-Fuzzy comprehensive evaluation," Nurse Education Today, vol. 77, pp. 77-82, 2019.

[24] J. Q. Zhang, X. B. Chen, and Q. B. Sun, “An assessment model of safety production management based on fuzzy comprehensive evaluation method and behavior-based safety," Mathematical Problems in Engineering, vol. 2019, Article ID 4137035, 11 pages, 2019.

[25] X. F. Zhu, Y. J. Wang, and D. J. Li, "The effectiveness test of the maximum membership principle in fuzzy comprehensive evaluation," Geomatics \& Spatial Information Technology, vol. 39, no. 5, pp. 136-137, 2016.

[26] H. L. Liu, L. S. Lu, J. W. Sun et al., "Manufacture of highquality chopped carbon fibers based on fuzzy comprehensive evaluation," Advances in Mechanical Engineering, vol. 9, no. 6, pp. 1-12, 2017.

[27] J. Wang and X. L. Dong, "Improvement the maximum membership degree principle in fuzzy evaluation," Hebei Water Conservancy, no. 2, pp. 27-28, 2011.

[28] L. Fan, "Research on variable rate spraying control system for boom-transformable sprayer," Master thesis, Nanjing Forestry University, Nanjing, China, 2016.

[29] L. X. Shi, "Design of boom-transformable sprayer with adjustable row spacing," Master thesis, Nanjing Forestry University, Nanjing, China, 2016.

[30] X. H. Xiao, "Influencing factors of pesticide utilization rate and its countermeasures," South China Agriculture, vol. 11, no. 7, pp. 5-7, 2017.

[31] Z. Y. Wen, "Experimental investigation of droplet characteristics and drift performance in air-assist boom sprayer," Master thesis, Jiangsu University, Zhenjiang, China, 2016.

[32] H. Lin, H. Zhou, L. Xu, H. Zhu, and H. Huang, "Effect of surfactant concentration on the spreading properties of pesticide droplets on Eucalyptus leaves," Biosystems Engineering, vol. 143, pp. 42-49, 2016. 